\title{
Computing the zeros of the partial sums of the Riemann zeta function $^{1}$
}

G. Mora and J.M. Sepulcre

Department of Mathematical Analysis, University of Alicante, 03080-Alicante, Spain.

E-mail address: JM.Sepulcre@ua.es

ABSTRACT In this paper we introduce a formula for the exact number of zeros of every partial sum of the Riemann zeta function inside infinitely many rectangles of the critical strips where they are situated.

AMS Subject Classification: 30Axx, 30Dxx.

Key Words: Zeros of entire functions, Exponential Polynomials, Partial Sums of the Riemann Zeta Function.

\section{Introduction}

The zeros of exponential polynomials is a topic which appeared in the first third of the twentieth century in relation to the development of the theory of differential equations. At this point we must quote Wilder [14]. Here it can be found one of the first formulae to determine the number of zeros of an exponential sum inside a rectangle of the critical strip where its zeros are located. On the line which Wilder had indicated are the works of Tamarkin $[11,12]$ and Langer $[6]$ on the zeros of certain functions in connection with some general problems of the expansion theory for linear differential equations (see [11, p. 66]). Analogous results can be found in [13], where Turan attributes to Pólya [10] a formula for the number of the zeros of an exponential sum where the coefficients are algebraic polynomials. Also, certain ideas considered by Tamarkin, Wilder and Langer were developed in the sixties by Dickson $[3,4]$.

Similar formulae on the number of zeros of functions much more general than exponential polynomials are provided in Levin's book [7]. For instance, if the spectrum of an almost-periodic function $f(z)$ is contained in the set of the vertexes of a segment of the imaginary axis, the formula is, for sufficiently large values of $\left|y_{1}\right|$ and $\left|y_{2}\right|$,

$$
N\left(x_{1}, x_{2}, y_{1}, y_{2}\right)=\frac{d}{2 \pi}\left(x_{2}-x_{1}\right)+O(1)
$$

where $d$ is the length of the segment and $N\left(x_{1}, x_{2}, y_{1}, y_{2}\right)$ denotes the number of zeros of $f(z)$ inside the rectangle $x_{1} \leq x \leq x_{2}, y_{1} \leq y \leq y_{2}$.

Recently, for the partial sums

$$
\zeta_{n}(s)=\sum_{k=1}^{n} k^{-s},
$$

\footnotetext{
${ }^{1}$ Preprint.
} 
with $s=\sigma+i t$ a complex variable and $n$ a real number greater than or equal to 2, Gonek and Ledoan prove in [5, Theorem 2] the formula

$$
\left|N_{n}(T)-\frac{T}{2 \pi} \log [n]\right|<\frac{n}{2},
$$

which determines the number of zeros $N_{n}(T)$ of $\zeta_{n}(s)$ for the special case when these zeros have ordinates in $[0, T]$. Here $[x]$ is used to denote the largest integer not exceeding the real number $x$. Therefore, it is worth to note that the term $O(1)$ of formula (1.1) may depend on $f(z)$ such as the result of Gonek-Ledoan makes explicit for the sequence of functions $\zeta_{n}(s)$.

As we have just seen, in the extensive literature on the related question with the topic of the zeros, the formulae for the number of zeros in certain regions, mainly rectangles, have a common thing: all them contain either the error term $O(1)$ or a bound which expresses the maximum error with respect to the exact number of zeros inside those regions. For the partial sums of the Riemann zeta function $\zeta_{n}(s)$, with $n \geq 2$ an integer, we established in [9, Theorem 6] a formula for the number of their zeros inside certain rectangles in the critical strip. In fact, we proved the existence of infinitely many rectangles $\left\{R_{n, T}\right\}$ bounded by the lines $y=0$ and $y=T, T>0$, such that the number of zeros, $N_{n}(T)$, of $\zeta_{n}(s)$ inside each $R_{n, T}$ satisfies

$$
N_{n}(T)=\left[\frac{T \log n}{2 \pi}+\Omega_{n}\right], \text { with }\left|\Omega_{n}\right|<1 .
$$

In this paper we deal with the problem of the existence of rectangles in the critical strip of every $\zeta_{n}(s)$ for which the error in the formula for the number of zeros can be reduced to 0 . In terms of the last paper of Gonek and Ledoan [5], the question would be expressed as follows: Are there rectangles in the critical strip given by the lines $y=0$ and $y=T$ for which the formula (1.2) is exact? We have just proved that the answer is yes; in fact, we demonstrate the existence of infinitely many values of $T$ where the zeros of every partial sum $\zeta_{n}(s)$ inside the corresponding rectangles can be counted by the formula

$$
N_{n}(T)=\left[\frac{T \log n}{2 \pi}\right] .
$$

It is worthwhile to remark that this result improves that of [9, Theorem 6].

\section{The formula}

For each integer $n \geq 2, \zeta_{n}(s)$ is an entire function of order 1, exponential type $\ln n$, and it has infinitely many zeros not all of them are situated on the imaginary axis, except for the case $n=2[9]$ whose zeros $z_{k}$ are explicitly given by

$$
z_{k}=\frac{(2 k+1) \pi i}{\ln 2}, \quad k \in \mathbb{Z} .
$$


Therefore, $n=2$ is the the trivial case, and we will assume that $n>2$.

On the other hand, since for any $t$ we have

$$
\lim _{\sigma \rightarrow-\infty} \zeta_{n}(\sigma+i t) n^{\sigma+i t}=1
$$

and

$$
\lim _{\sigma \rightarrow \infty} \zeta_{n}(\sigma+i t)=1,
$$

there exist two values of $\sigma, \sigma_{n_{1}}<0<\sigma_{n_{2}}$, such that

$$
\left|\zeta_{n}(s) n^{s}-1\right|<1 \text { for all } s \text { with } \operatorname{Re} s \leq \sigma_{n_{1}}
$$

and

$$
\left|\zeta_{n}(s)-1\right|<1 \text { for all } s \text { with } \operatorname{Re} s \geq \sigma_{n_{2}} .
$$

Therefore the functions $\zeta_{n}(s)$ have all their zeros comprised in vertical strips $S_{n}$, called critical strips, defined by

$$
S_{n}:=\left\{s=\sigma+i t: a_{n} \leq \sigma \leq b_{n}\right\},
$$

where the bounds

$$
a_{n}:=\inf \left\{\operatorname{Re} s: \zeta_{n}(s)=0\right\}
$$

and

$$
b_{n}:=\sup \left\{\operatorname{Re} s: \zeta_{n}(s)=0\right\}
$$

have been estimated by means of the expressions

$$
-n \log 2+o(1)
$$

and

$$
1+\left(\frac{4}{\pi}-1+o(1)\right) \frac{\log \log n}{\log n}
$$

by Balazard and Velazquez-Castañon [2] and Montgomery [8], respectively.

Remark 1 We first take a rectangle, denoted by $R_{n, T}$, defined by the right-lines $x=a_{n}^{\prime}, x=b_{n}^{\prime} ; y=0$ and $y=T$, where $a_{n}^{\prime}$ and $b_{n}^{\prime}$ are arbitrary real numbers satisfying $a_{n}^{\prime}<a_{n}, b_{n}^{\prime}>b_{n}$, and $T>0$ is so that $\zeta_{n}(s)$ has no zero on the line $y=T$. The values of $T$ for which formula (1.3) is valid will be specified in the proof of the next theorem.

Theorem 2 For every integer $n \geq 2$, there exist infinitely many rectangles $R_{n, T}$ such that the number of zeros, $N_{n}(T)$, of the function $\zeta_{n}(s)$ inside each one of them is given by the formula

$$
N_{n}(T)=\left[\frac{T \log n}{2 \pi}\right] .
$$


Proof. For $n=2$, we consider a rectangle $R_{2, T}$ with $T \in\left[\frac{2 \pi k}{\log 2}, \frac{\pi(2 k+1)}{\log 2}\right)$ for some integer $k \geq 0$. Then $k \leq \frac{T \log 2}{2 \pi}<k+\frac{1}{2}$, therefore $\left[\frac{T \log 2}{2 \pi}\right]=k$. Noticing (2.1) the number of zeros inside $R_{2, T}$ satisfies

$$
N_{2}(T)=k,
$$

and this means that the formula (1.3) follows. Therefore, from now on we assume that $n>2$. Consider a rectangle $R_{n, T}$; we observe that $\zeta_{n}(s)$ is never 0 on the boundary of $R_{n, T}$, therefore we can apply the argument principle [1, p. 87] on it. For a given $0<\epsilon<\frac{1}{6}$, by virtue of (2.2) and (2.3), we determine two values $a_{n}^{\prime \prime}, b_{n}^{\prime \prime}$, with $a_{n}^{\prime \prime}<a_{n}^{\prime}$ and $b_{n}^{\prime \prime}>b_{n}^{\prime}$, satisfying

$$
\left|\zeta_{n}(s) n^{s}-1\right|<\frac{1}{6} \sin \epsilon \text { for all } s \text { with } \operatorname{Re} s=a_{n}^{\prime \prime},
$$

and

$$
\left|\zeta_{n}(s)-1\right|<\frac{1}{6} \sin \epsilon \text { for all } s \text { with } \operatorname{Re} s=b_{n}^{\prime \prime} .
$$

Let $R_{n, T}^{\prime}$ be a new rectangle defined by the right-lines $x=a_{n}^{\prime \prime}, x=b_{n}^{\prime \prime} ; y=0$, $y=T$. Noticing $\operatorname{Re} s \leq a_{n}^{\prime}$ and $\operatorname{Re} s \geq b_{n}^{\prime}$ are zero-free regions, we conclude that $\zeta_{n}(s)$ has the same number of zeros inside both rectangles $R_{n, T}$ and $R_{n, T}^{\prime}$. Then, for the above $\epsilon$, we claim that there exist infinitely many values of $T$ such that the variation of the argument of $\zeta_{n}(s)$ on the boundary of $R_{n, T}^{\prime}$, denoted by $V A\left(\zeta_{n}(s) ; R_{n, T}^{\prime}\right)$, satisfies

$$
V A\left(\zeta_{n}(s) ; R_{n, T}^{\prime}\right)=T \log n+\theta, \text { with }|\theta|<\epsilon .
$$

Indeed, by writing

$$
\zeta_{n}(s)=e^{-s \log n}\left(\zeta_{n}(s) n^{s}\right),
$$

and according to (2.4), we infer that the variation of the argument of $\zeta_{n}(s)$ on the side of the rectangle $R_{n, T}^{\prime}$ defined by the line $x=a_{n}^{\prime \prime}$, denoted by $V A\left(\zeta_{n}(s) ; x=\right.$ $\left.a_{n}^{\prime \prime}\right)$, is given by

$$
V A\left(\zeta_{n}(s) ; x=a_{n}^{\prime \prime}\right)=T \log n+\alpha, \text { with }|\alpha|<\frac{\epsilon}{3} .
$$

Now, from (2.5), we conclude that the variation of the argument of $\zeta_{n}(s)$ on the side of the rectangle $R_{n, T}^{\prime}$ defined by the line $x=b_{n}^{\prime \prime}$, denoted by $V A\left(\zeta_{n}(s) ; x=b_{n}^{\prime \prime}\right)$, satisfies

$$
\left|V A\left(\zeta_{n}(s) ; x=b_{n}^{\prime \prime}\right)\right|<\frac{\epsilon}{3} .
$$

On the other hand, because $\zeta_{n}(s)>0$ for all real $s$, the variation of the argument of $\zeta_{n}(s)$ on the side of the rectangle $R_{n, T}^{\prime}$ defined by the line $y=0$, denoted by $V A\left(\zeta_{n}(s) ; y=0\right)$, is

$$
V A\left(\zeta_{n}(s) ; y=0\right)=0 .
$$


Finally, it only remains to prove that there exist infinitely many values of $T$ such that the variation of the argument of $\zeta_{n}(s)$ on the side of the rectangle $R_{n, T}^{\prime}$ defined by the line $y=T, V A\left(\zeta_{n}(s) ; y=T\right)$, satisfies

$$
\left|V A\left(\zeta_{n}(s) ; y=T\right)\right|<\frac{\epsilon}{3} .
$$

Indeed, let $\left\{p_{1}, p_{2}, \ldots, p_{k_{n}}\right\}$ be the set of all prime numbers less than or equal to $n$; noticing for each integer $1 \leq m \leq n$ there exist non-negative integers $l_{m j}$ such that

$$
\log m=\sum_{j=1}^{k_{n}} l_{m j} \log p_{j},
$$

the functions $\operatorname{Re} \zeta_{n}(s)$ and $\operatorname{Im} \zeta_{n}(s)$ can be considered as polynomial in

$$
e^{\sigma \ln p_{j}}, \cos \left(t \log p_{j}\right), \sin \left(t \log p_{j}\right), j=1, \ldots, k_{n} .
$$

Given the preceding $\epsilon$, by continuity, there exists $\delta>0$ such that for any real number $\sigma \in\left[a_{n}^{\prime \prime}, b_{n}^{\prime \prime}\right]$ one has

$$
\left|\operatorname{Re} \zeta_{n}(\sigma+i t)-\operatorname{Re} \zeta_{n}(\sigma)\right|<\epsilon
$$

provided that

$$
\left|\cos \left(t \log p_{j}\right)-1\right|<\delta,\left|\sin \left(t \log p_{j}\right)\right|<\delta
$$

for all $j=1, \ldots, k_{n}$. Given $\delta>0$, determine a positive number $\eta<\epsilon$ so that for any $\eta_{j}$ satisfying $\left|\eta_{j}\right| \leq \eta$, the conditions (2.13) to be fulfilled, that is

$$
\left|\cos \left(\eta_{j} \log p_{j}\right)-1\right|<\delta,\left|\sin \left(\eta_{j} \log p_{j}\right)\right|<\delta, \text { for all } j=1, \ldots, k_{n} .
$$

Now, since for any $s=\sigma+i t$

$$
\operatorname{Im} \zeta_{n}(s)=-e^{-\sigma \log 2} \sin (t \log 2)-\ldots-e^{-\sigma \log n} \sin (t \log n),
$$

by using (2.11) we can write, for $\sigma \in\left[a_{n}^{\prime \prime}, b_{n}^{\prime \prime}\right]$,

$$
\operatorname{Im} \zeta_{n}(s)=\sum_{j=1}^{k_{n}} \sin \left(t \log p_{j}\right) f_{j}(t, \sigma),
$$

where $f_{j}(t, \sigma)$ is a uniformly bounded function on $\mathbb{R} \times\left[a_{n}^{\prime \prime}, b_{n}^{\prime \prime}\right]$. Let $M>0$ be such that $\left|f_{j}(t, \sigma)\right| \leq M$ for all $j=1, \ldots, k_{n}$. Define a positive number

$$
\lambda=\min \left\{\frac{\eta}{\log p_{k_{n}}}, \frac{\frac{5}{36} \epsilon}{M \log \left(p_{1} \ldots p_{k_{n}}\right.}\right\}
$$

and let us take

$$
\alpha_{j}=\frac{2 \pi}{\ln p_{j}}, \beta_{j}=0,1 \leq j \leq k_{n} \text {, and } \lambda .
$$


Then, as the numbers $\frac{1}{\alpha_{j}}$ are linearly independent over the rationals, by applying a lemma of Kronecker and Bohl [11, p. 68], there exists a positive real number $l$ such that any real interval of the form

$$
(p l,(p+1) l), p \in \mathbb{Z},
$$

contains an interval $I_{p}$ of length $\lambda$ which contains at least one point of each set

$$
A_{j}:=\left\{\frac{2 \pi q}{\log p_{j}}: q \in \mathbb{Z}\right\}, 1 \leq j \leq k_{n} .
$$

Let $T$ be an arbitrary point of some $I_{p}$ with $p \geq 0$. For each $j \in\left\{1,2, \ldots, k_{n}\right\}$ determine $\gamma_{j} \in I_{p} \cap A_{j}$ and define $\eta_{j}=T-\gamma_{j}$. Since $p_{k_{n}} \geq 3$, because (2.16) we can assure that $\left|\eta_{j}\right| \leq \eta$, and according to each $\gamma_{j}=\frac{2 \pi q}{\log p_{j}}$, with $q$ integer, by substituting the values of $\eta_{j}$ into (2.14), we get

$$
\left|\cos \left(T \log p_{j}\right)-1\right|<\delta,\left|\sin \left(T \log p_{j}\right)\right|<\delta, \text { for all } 1 \leq j \leq k_{n},
$$

and, consequently, (2.12) is true for $t=T$. Then, according to $\operatorname{Re} \zeta_{n}(\sigma)>1$ for all $\sigma \in \mathbb{R}$, one has

$$
\operatorname{Re} \zeta_{n}(\sigma+i T)=\operatorname{Re} \zeta_{n}(\sigma)+\left(\operatorname{Re} \zeta_{n}(\sigma+i T)-\operatorname{Re} \zeta_{n}(\sigma)\right) \geq 1-\epsilon,
$$

for all $\sigma \in\left[a_{n}^{\prime \prime}, b_{n}^{\prime \prime}\right]$.

About the imaginary part of $\zeta_{n}(s)$, by setting $s=\sigma+i T$ with $\sigma \in\left[a_{n}^{\prime \prime}, b_{n}^{\prime \prime}\right]$ in $(2.15)$, because of $(2.16)$, we have

$$
\begin{aligned}
\left|\operatorname{Im} \zeta_{n}(\sigma+i T)\right| \leq & M \sum_{j=1}^{k_{n}}\left|\sin \left(\eta_{j} \log p_{j}\right)\right| \leq M \sum_{j=1}^{k_{n}}\left|\eta_{j} \log p_{j}\right| \leq \\
& \leq M \lambda \log \left(p_{1} \ldots p_{k_{n}}\right) \leq \frac{5}{36} \epsilon .
\end{aligned}
$$

From (2.17) and (2.18), it deduces that the variation of the argument of $\zeta_{n}(s)$ on the side of the rectangle $R_{n, T}^{\prime}$ defined by the line $y=T, V A\left(\zeta_{n}(s) ; y=T\right)$, satisfies

$$
V A\left(\zeta_{n}(s) ; y=T\right) \leq 2 \arctan \left(\frac{\frac{5}{36} \epsilon}{1-\epsilon}\right)
$$

Now, recalling that we have chosen $\epsilon$ so that $0<\epsilon<\frac{1}{6}$ and taking into account that $\arctan x \leq x$, for all $x \in \mathbb{R}$, the above inequality implies that

$$
V A\left(\zeta_{n}(s) ; y=T\right) \leq \frac{\frac{5}{18} \epsilon}{1-\epsilon}<\frac{\epsilon}{3}
$$

and then (2.10) is true, as claimed. Now, according to (2.7), (2.8), (2.9) and (2.10), it follows (2.6) for any point $T$ of every $I_{p}$ with $p \geq 0$ and, in consequence, the formula (1.3) is true. 


\section{References}

[1] Ash, R.B.: Complex Variables, Academic Press, New York (1971)

[2] Balazard, M., Velásquez-Castañón, O.: Sur l'infimum des parties réelles des zéros des sommes partielles de la fonction zêta de Riemann, C. R. Acad. Sci. Paris, Ser. I 347, 343-346 (2009)

[3] Dickson, D. G.: Asymptotic distribution of zeros of exponential sums, Publ. Math. Debrecen 11, 297-300 (1964)

[4] Dickson, D. G.: Zeros of exponential sums, Proc. of the Amer. Math. Soc., Vol. 16, No. 1, 84-89 (1965)

[5] Gonek, S.M., Ledoan, A.H.: Zeros of partial sums of the Riemann Zetafunction, Int. Math. Res. Not. 10, 1775-1791 (2010)

[6] Langer, R. E.: On the zeros of exponential sums and integrals, Bull. Amer. Math. Soc. 37, 213-239 (1931)

[7] Levin, B. J.: Distribution of Zeros of Entire Functions, Amer. Math. Soc., Providence (1980)

[8] Montgomery, H.L.: Zeros of approximations to the zeta function, Studies in Pure Mathematics: to the memory of Paul Turán, Birkhäuser, Basel, pp. 497-506 (1983)

[9] Mora, G., Sepulcre, J.M.: On the distribution of zeros of a sequence of entire functions approaching the Riemann zeta function, J. Math. Anal. Appl. 350, 409-415 (2009)

[10] Pólya, G.: Jber. Deutch. Math. Verein, 3, p. 97, Problem 24 (1925)

[11] Tamarkin, J. D.: The zeros of certain integral functions, J. London Math. Soc. 2, 66-69 (1927)

[12] Tamarkin, J. D.: Some general problems of the theory of ordinary linear differential equations and expansions of an arbitrary function in series of fundamental functions, Math. Z. 27, 1-54 (1928)

[13] Turán, P.: On some approximative Dirichlet-polynomials in the theory of the zeta-function of Riemann, Danske Vid. Selsk. Mat.-Fys. Medd., 24, no. $17,3-36(1948)$

[14] Wilder, C. E.: Expansion problems of ordinary linear differential equations with auxiliary conditions at more than two points, Trans. Amer. Math. Soc. 18, 415-442 (1917) 